\title{
Extended Schatten norms of random graphs and Nikiforov conjecture
}

\author{
Ivan Lazarević(D) \\ Bulevar kralja Aleksandra 73, 11000, Belgrade
}

\begin{abstract}
In this paper we give mean of $p$-th degree of singular values and upper bound of geometric mean for almost all graphs. We prove three theorems about a conjecture of V. Nikiforov for Schatten $p$-norm of graphs when $p>2$. We prove that the conjecture is true when $p$ is an even integer or when graph is a tree or a strongly regular graph with certain parameters. The strongly regular graphs with these parameters are graphs with maximal energy.
\end{abstract}

Mathematics Subject Classification (2020). 15A18, 15A60, 05C80

Keywords. singular values of graph, Schatten norms, random graph, energy of graph

\section{Introduction}

In this paper we consider the undirected graph $G$ without loops. A tree is a conected graph without loops which has $n$ vertices and $n-1$ edges. In 1978. Ivan Gutman introduced the energy of graphs, as a sum of absolute values of eigenvalues of adjacency matrix $A$ of graph $G$. The singular values $\sigma_{1} \geq \sigma_{2} \geq \ldots \geq \sigma_{n}$ of adjacency matrix $A$ of graph $G$ are the square roots of eigenvalues of $A \cdot A^{T}$. Koolen and Moulton in [4] proved the following inequality about the maximal energy of a graph:

$$
E(G) \leq \frac{n \sqrt{n}}{2}+\frac{n}{2}
$$

and the equality holds when $G$ is a strongly regular graph with parameters

$$
\left(n, \frac{n+\sqrt{n}}{2}, \frac{n+2 \sqrt{n}}{4}, \frac{n+2 \sqrt{n}}{4}\right) .
$$

A strongly regular graph with parameters $(n, k, \lambda, \mu)$ is a regular graph of degree $k$ (number of neighbours for every vertex), $\lambda$ is a number of common neighbours of adjacent vertices and $\mu$ number of common neighbours of non-adjacent vertices.

V. Nikiforov proved in [6] that energy of almost all graphs is:

$$
E(G)=\left(\frac{4}{3 \pi}+o(1)\right) n^{\frac{3}{2}}
$$

In section 2 of this paper we give an overview of the main features of random matrices and Wigner's semicircle law and we calculate the mean of $p$-th degree of singular values

Email address: ilazarevic@grf.bg.ac.rs

Received: 12.04.2021; Accepted: 22.01.2022 
of almost all graphs and an upper bound for the geometric mean of singular values of random graphs. An adjacency matrix of a random graph is a random matrix, that is a matrix whose elements are random variables.

Now, we state definitions of two classes of norms.

Definition 1.1. Let $p \geq 1$ be a real number and $n \geq 1$. If $A \in M_{n, n}$ is an adjacency matrix of a graph $G$ and $\sigma_{1} \geq \sigma_{2} \geq \ldots \geq \sigma_{n}$ are the singular values of $A$, the Schatten p-norm $\|A\|_{p}$ is given by

$$
\|A\|_{p}=\left(\sigma_{1}^{p}+\sigma_{2}^{p}+\ldots+\sigma_{n}^{p}\right)^{\frac{1}{p}}
$$

Definition 1.2. Let $n \geq 1$ and $A \in M_{n, n}$ be an adjacency matrix of graph $G$ and $\sigma_{1} \geq \sigma_{2} \geq \ldots \geq \sigma_{n}$ are the singular values of $A$, the Ky Fan k-norm $\|A\|_{k}$ is given by

$$
\|A\|_{k}=\sigma_{1}+\sigma_{2}+\ldots+\sigma_{k}
$$

V. Nikiforov calculated Schatten norm for almost all graphs in three cases: $1 \leq p<2$, $p=2$ and $p>2$ in Theorem 5 in [7]. In section 2 we calculate a mean of $p$-th degree of singular values of almost all graphs; this is a generalization of Schatten norm result for $0<p<1$. When $p=0$ we get a determinant of a random graph. When $p=1$ we get the energy of a graph. The problem of maximal energy of a graph is very important. The problem of maximal determinant has great application in theory of optimal design, see $[2,5,9]$. In section 3 , we prove three theorems about the conjecture of V. Nikiforov from [8]. We proved that a complete graph has larger Schatten norm (when $p>2$ ) than a tree, a strongly regular graph with parameters $\left(n, \frac{n+\sqrt{n}}{2}, \frac{n+2 \sqrt{n}}{4}, \frac{n+2 \sqrt{n}}{4}\right)$, and any undirected graph when $p$ is an even integer.

\section{Random graphs}

The eigenvalues of random matrices have Wigner's semicircle distribution, see $[1,10]$. Let $A$ be an adjacency matrix of a graph $G, A=\left[a_{i j}\right]_{n \times n}$, a symmetric one-zero matrix with zeroes on main diagonal. The entries $a_{i j}\left(a_{i j}=a_{j i}\right.$ and $\left.1 \leq i \leq j \leq n\right)$ are independent random variables with values 0 and 1 with probability $\frac{1}{2}$, and $a_{i i}=0$ with probability 1 . Thus, the mathematical expectation and dispersion are: $E\left(a_{i j}\right)=\frac{1}{2}, D\left(a_{i j}\right)=\sigma^{2}=\frac{1}{4}$. Let the distribution function for $a_{i j}$ be $F$. We consider a normalized matrix:

$$
B=\frac{1}{2 \sigma \sqrt{n}} A=\frac{1}{\sqrt{n}} A
$$

with eigenvalues $\lambda_{1}, \ldots, \lambda_{n}$. Let $N_{n}(x)$ be a number of eigenvalues with values less than $x$. Let the function $W_{n}(x)=\frac{N_{n}(x)}{n}$ be an empirical distribution function for $\lambda_{1}, \ldots, \lambda_{n}$. The next theorem is an important result from [10].

Theorem 2.1. Under the following conditions:

(1) $\int|x|^{p} d F<\infty$, for all $p$.

(2) The distribution function $F$ is symmetric (all odd moments are equal to zero). Then $W_{n}(x)$ converge in distribution to a random variable $W(x)$, where $W(x)$ is Wigner's semicircle continuous distribution function.

Here, the density of Wigner's semicircle continuous distribution is:

$$
w(x)= \begin{cases}\frac{2}{\pi} \sqrt{1-x^{2}}, & |x| \leq 1 \\ 0, & |x|>1\end{cases}
$$


U. Grenander in [3] proved that under the conditions from this theorem

$$
\lim _{n \rightarrow \infty} W_{n}(x)=W(x)
$$

in probability.

L. Arnold in [1] proved the following theorem:

Theorem 2.2. (1) If $\int x^{4} d F<\infty$ and $\int x d F=0$ then for empirical distribution function $W_{n}(x)$ the following holds:

$$
\lim _{n \rightarrow \infty} W_{n}(x)=W(x)
$$

in probability.

(2) If $\int x^{6} d F<\infty$ then

$$
\lim _{n \rightarrow \infty} W_{n}(x)=W(x)
$$

almost surely.

Remark 2.3. The previous theorems are given in this paper in special cases that can be applied to adjacency matrix of a random graph $\left(a_{i i}=0\right)$. Originally these theorems refer to any random matrix and have additional conditions, see [1].

The following theorem was proved with essentially the same argument in V. Nikiforov's paper [6]. We just extend it slightly to include cases when $0<p<1$ by considering the mean of $p$-th degree of singular values instead of the norm.

Theorem 2.4. The mean of $p$-th degrees of singular values of almost all graphs has the following value:

$$
M_{\lambda}=\left(\frac{2}{\pi} B\left(\frac{p+1}{2}, \frac{3}{2}\right)+o(1)\right)^{\frac{1}{p}} \sqrt{n}
$$

Where $B\left(\frac{p+1}{2}, \frac{3}{2}\right)$ is the beta function with parameters $\frac{p+1}{2}$ and $\frac{3}{2}$.

Proof. The sum of $p$-th degrees of absolute values of eigenvalues of almost all graphs is the $p$-th moment of a random variable. Consequently we calculate the following integral:

$$
\int_{0}^{1} x^{p} \sqrt{1-x^{2}} d x=\frac{1}{2} \int_{0}^{1} \sqrt{1-t} \cdot t^{\frac{p-1}{2}} d t=\frac{1}{2} B\left(\frac{p+1}{2}, \frac{3}{2}\right)
$$

Everything is multiplied by $\sqrt{n}$ because $\lambda_{1}, \ldots, \lambda_{n}$ are eigenvalues of matrix $B=\frac{1}{\sqrt{n}} A$.

Theorem 2.5. The value of the geometric mean of singular values of almost all graphs with $n$ vertices is less or equal to

$$
\sqrt{\frac{n}{4 e}}(1+o(1))
$$

Proof.

$$
\sqrt[n]{\sigma_{1} \cdot \sigma_{2} \cdots \sigma_{n}}=e^{\frac{1}{n} \ln \left(\sigma_{1} \cdot \sigma_{2} \cdots \sigma_{n}\right)}=e^{\frac{1}{n} \sum_{k=1}^{n} \ln \sigma_{k}}
$$

From paper [1] (see Theorems 1 and 2 above), it follows:

$$
\frac{1}{n} \sum_{k=1}^{n} \ln \frac{\sigma_{k}}{\sqrt{n}} \leq \frac{4}{\pi} \int_{0}^{1} \ln x \sqrt{1-x^{2}} d x+o(1)
$$

Now, we calculate that integral:

$$
I=\int_{0}^{1} \ln x \sqrt{1-x^{2}} d x
$$


Notice that:

$$
\int_{0}^{1} \ln x \sqrt{1-x^{2}} d x+\int_{0}^{1} \sqrt{1-x^{2}} d x=\int_{0}^{1}(1+\ln x) \sqrt{1-x^{2}} d x
$$

And we calculate the last integral using the method of partial integration:

$$
\begin{gathered}
\int_{0}^{1}(1+\ln x) \sqrt{1-x^{2}} d x=-\int_{0}^{1} \frac{-x^{2} \ln x}{\sqrt{1-x^{2}}} d x=-I+\int_{0}^{1} \frac{\ln x}{\sqrt{1-x^{2}}} d x \\
2 I+\int_{0}^{1} \sqrt{1-x^{2}} d x=\int_{0}^{1} \frac{\ln x}{\sqrt{1-x^{2}}} d x
\end{gathered}
$$

We label the last integral $I_{1}$ and calculate this:

Now, using substitution:

$$
I_{1}=\int_{0}^{1} \frac{\ln x}{\sqrt{1-x^{2}}} d x=\int_{0}^{\frac{\pi}{2}} \ln \sin t d t
$$

$$
\begin{gathered}
\int_{0}^{a} f(a-x) d x=\int_{0}^{a} f(x) d x \Rightarrow \\
\Rightarrow \int_{0}^{\frac{\pi}{2}} \ln \sin t d t=\int_{0}^{\frac{\pi}{2}} \ln \cos t d t \\
2 I_{1}=\int_{0}^{\frac{\pi}{2}} \ln \sin t d t+\int_{0}^{\frac{\pi}{2}} \ln \cos t d t=\int_{0}^{\frac{\pi}{2}} \ln \sin 2 t d t-\int_{0}^{\frac{\pi}{2}} \ln 2 d t \\
I_{1}=-\frac{\pi}{2} \ln 2 \Rightarrow I=-\frac{\pi}{8}-\frac{\pi}{4} \ln 2 \Rightarrow \\
\Rightarrow \frac{1}{n} \sum_{k=1}^{n} \ln \frac{\sigma_{k}}{\sqrt{n}} \leq-\frac{1}{2}-\ln 2+o(1) \Leftrightarrow e^{\frac{1}{n} \sum_{k=1}^{n} \ln \sigma_{k}} \leq e^{\ln \sqrt{n}-\frac{1}{2}-\ln 2+o(1)}
\end{gathered}
$$

The initial statement clearly follows from this inequality.

\section{Maximum Schatten norms}

The Nikiforov conjecture (Conjecture 4.28 from [8]) is that for $p>2$ the maximal Shatten norm graph is the complete graph. The spectrum of a complete graph with $n$ vertices is: $\lambda_{1}=n-1, \lambda_{2}=\lambda_{3}=\ldots=\lambda_{n}=-1$.

Theorem 3.1. Let $G$ be undirected graph with $n$ vertices, $\sigma_{1} \geq \sigma_{2} \geq \ldots \geq \sigma_{n}$ be the singular values of $G$ and $p>2$ an even integer, then:

$$
\sigma_{1}^{p}+\sigma_{2}^{p}+\ldots .+\sigma_{n}^{p} \leq(n-1)^{p}+n-1
$$

Proof. Let $A$ be an adjacency matrix of a graph $G$ and $p>2$ be an even integer. The value of element $a_{i j}$ of matrix $A^{p}$ is given as a number of paths of length $p$ from vertex $i$ to vertex $j$. If $\lambda$ is an eigenvalue of matrix $A$ then $\lambda^{p}$ is an eigenvalue of matrix $A^{p}$, and their sum is the trace. The number of paths is decreasing when we reduce the number of edges. So it is clear, from these simple observations, that the inequality is true.

When $p=1$ the maximum Schatten norm is equal to the maximal energy of a graph and this is achieved for strongly regular graphs with parameters $\left(n, \frac{n+\sqrt{n}}{2}, \frac{n+2 \sqrt{n}}{4}, \frac{n+2 \sqrt{n}}{4}\right)$, where $n=4^{k}$. It is interesting to check if the Nikiforov conjecture is true for the previously mentioned graphs. The answer to this question is given by the following theorem. 
Theorem 3.2. Let $G$ be a strongly regular graph with parameters $\left(n, \frac{n+\sqrt{n}}{2}, \frac{n+2 \sqrt{n}}{4}, \frac{n+2 \sqrt{n}}{4}\right)$ with the singular values $\sigma_{1} \geq \sigma_{2} \geq \ldots \geq \sigma_{n}$, where $n=4^{k}$, then:

$$
\sigma_{1}^{p}+\sigma_{2}^{p}+\ldots .+\sigma_{n}^{p} \leq(n-1)^{p}+n-1
$$

Proof. We prove this theorem using mathematical induction.

Let $n=4^{k}$. We know that singular values of a regular graph with these parameters are: $\sigma_{1}=\frac{2^{k}+4^{k}}{2} ; \sigma_{2}=\sigma_{3}=\ldots=\sigma_{n}=\frac{2^{k}}{2}$. In this notation, the inequality to be proved becomes:

$$
2^{k p}\left(\left(2^{k}+1\right)^{p-1}+2^{k}-1\right) \leq 2^{p}\left(2^{k}-1\right)\left(\left(4^{k}-1\right)^{p-1}+1\right)
$$

When $k=1$, i.e. for $n=4$, a strongly regular graph is a complete graph so the equality holds. Suppose now that the inequality is true for $k$ and let us prove it for $k+1$.

We have to prove:

$$
2^{(k+1) p}\left(\left(2 \cdot 2^{k}+1\right)^{p-1}+2 \cdot 2^{k}-1\right) \leq 2^{p}\left(2^{k+1}-1\right)\left(\left(4^{k+1}-1\right)^{p-1}+1\right)
$$

Now, we consider the left side of inequality:

$$
\begin{aligned}
2^{k p} \cdot 2^{p}\left(\left(2 \cdot 2^{k}+1\right)^{p-1}+2 \cdot 2^{k}-1\right)=2^{k p} \cdot 2^{p}\left(2^{p-1}\left(2^{k}+\frac{1}{2}\right)^{p-1}+2 \cdot\left(2^{k}-\frac{1}{2}\right)\right) \\
\quad=2^{k p} \cdot 2^{p+1}\left(2^{p-2}\left(2^{k}+\frac{1}{2}\right)^{p-1}+2^{k}-\frac{1}{2}\right) \\
\leq 2^{k p} \cdot 2^{p+1} \cdot 2^{p-2}\left(\left(2^{k}+\frac{1}{2}\right)^{p-1}+2^{k}-\frac{1}{2}\right) \\
\leq 2^{k p} \cdot 2^{p+1} \cdot 2^{p-2}\left(\left(2^{k}+1\right)^{p-1}+2^{k}-1\right)
\end{aligned}
$$

In the last inequality we use the fact that the function $y=a^{x}-b^{x}$ is growing in $x$ when $a>b$.

Now, we apply the induction hypothesis:

$$
\begin{aligned}
& 2^{k p} \cdot 2^{p+1} \cdot 2^{p-2}\left(\left(2^{k}+1\right)^{p-1}+2^{k}-1\right) \\
\leq & 2^{p} \cdot 2^{p+1} \cdot 2^{p-2}\left(2^{k}-1\right)\left(\left(4^{k}-1\right)^{p-1}+1\right) \\
& =2^{p} \cdot 4^{p-1}\left(2^{k+1}-2\right)\left(\left(4^{k}-1\right)^{p-1}+1\right) \\
= & 2^{p} \cdot\left(2^{k+1}-2\right)\left(\left(4^{k+1}-4\right)^{p-1}+4^{p-1}\right) \\
& \leq 2^{p} \cdot\left(2^{k+1}-1\right)\left(\left(4^{k+1}-1\right)^{p-1}+1\right)
\end{aligned}
$$

Let us explain the inequality which we applied:

$$
\left(\left(4^{k+1}-4\right)^{p-1}+4^{p-1}\right) \leq\left(\left(4^{k+1}-1\right)^{p-1}+1\right)
$$

Consider following expression:

$$
\left(\left(4^{k+1}-1\right)^{p-1}+1\right)-\left(\left(4^{k+1}-4\right)^{p-1}+4^{p-1}\right)
$$

Let $4^{k+1}-1=a$ and $p-1=x$ then $a>63$ and $x>1$, the previous expression becomes:

$$
a^{x}+1-(a-3)^{x}-4^{x}=a^{x}-(a-3)^{x}-\left(4^{x}-(4-3)^{x}\right)
$$

The last expression is a difference of two values of a function $a^{x}-(a-3)^{x}$, which is growing in $a$ and positive, from which this it clearly follows that the considered inequality is true.

This completes the proof. 
Theorem 3.3. Let is $G$ a tree and $\sigma_{1} \geq \sigma_{2} \geq \ldots \geq \sigma_{n}$ the singular values of graph $G$ then:

$$
\sigma_{1}^{p}+\sigma_{2}^{p}+\ldots .+\sigma_{n}^{p} \leq(n-1)^{p}+n-1
$$

Proof. The important classical inequality for the largest eigenvalue of a tree is:

$$
\lambda_{1} \leq \sqrt{n-1}
$$

Thus, we have

$$
\sigma_{1}^{p}+\sigma_{2}^{p}+\ldots .+\sigma_{n}^{p} \leq(n-1)^{\frac{p}{2}}+\sigma_{2}^{p}+\sigma_{3}^{p}+\ldots \sigma_{n}^{p} \leq n(n-1)^{\frac{p}{2}}
$$

When $n>2, n \in N$ and $p>2$ the following inequality is true:

$$
n \leq(n-1)^{\frac{p}{2}}+(n-1)^{1-\frac{p}{2}}
$$

Therefore,

$$
n(n-1)^{\frac{p}{2}} \leq(n-1)^{p}+n-1
$$

The theorem is now proved.

Acknowledgment. The author is grateful to the editor and the referee(s) for their useful suggestions for improving the paper. Also, I wish to thank Professor Vladimir Božin for motivation and discussions during the preparation of the manuscript.

\section{References}

[1] L. Arnold, On the asymptotic distribution of the eigenvalues of random matrices, J. Math. Anal. Appl. 20, 262-268, 1967.

[2] T. Chadjipantelis, S. Kounias and C. Moyssiadis, The maximum determinant of $21 \times$ $21(+1,-1)$-matrices and D-optimal designs, J. Statist. Plan. Inference, 16, 167-178, 1987.

[3] U. Grenander, Probability on algebraic structures, Wiley, New York, 1963.

[4] J.H. Koolen and V. Moulton, Maximal energy graphs, Adv. Appl. Math. 26, 47-52, 2001.

[5] N. Nguyen and A.J. Miller, A review of some exchange algorithms for constructing discrete D-optimal designs, Comput. Stat. Data Anal. 14, 489-498, 1992.

[6] V. Nikiforov, The energy of graphs and matrices, J. Math. Anal. Appl. 326, 1472$1475,2007$.

[7] V. Nikiforov, Extremal norms of graphs and matrices, J. Math. Sci. 182, 164-174, 2012.

[8] V. Nikiforov, Beyond graph energy: Norms of graphs and matrices, Linear Algebra Appl. 506, 82-138, 2016.

[9] R. Sitter and B. Torsney, Optimal designs for binary response experiments with two design variables, Statist. Sinica, 5, 405-419, 1995.

[10] E. Wigner, On the distribution of the roots of certain symmetric matrices, Ann. of Math. (2) 67, 325-327, 1958. 